\title{
Periodic Solutions for Neutral Nonlinear Difference Equations with Functional Delay
}

\author{
Imene Soualhia, Abdelouaheb Ardjouni, \\ AND AHCENE DJOUdi
}

\begin{abstract}
We use a variant of Krasnoselskii's fixed point theorem to show that the nonlinear difference equation with functional delay

$$
\Delta x(t)=-a(t) g(x(t))+c(t) \Delta x(t-\tau(t))+q(t, x(t), x(t-\tau(t))),
$$

has periodic solutions. For that end, we invert this equation to construct a fixed point mapping written as a sum of a completely continuous map and a large contraction which is suitable for the application of Krasnoselskii-Burton's theorem.
\end{abstract}

\section{INTRODUCTION}

In this paper, we are interested in the analysis of qualitative theory of periodic solutions of difference equation. Motivated by the papers [1]-[13] and the references therein, we consider the following totally nonlinear difference equation with functional delay

$$
\begin{aligned}
\Delta x(t)= & -a(t) g(x(t))+c(t) \Delta x(t-\tau(t)) \\
& +q(t, x(t), x(t-\tau(t))), \quad t \in \mathbb{Z},
\end{aligned}
$$

where

$$
a, c: \mathbb{Z} \rightarrow \mathbb{R}, q: \mathbb{Z} \times \mathbb{R} \times \mathbb{R} \rightarrow \mathbb{R},
$$

with $\mathbb{Z}$ is the set of integers and $\mathbb{R}$ is the set of real numbers. Throughout this paper $\Delta$ denotes the forward difference operator

$$
\Delta x(t)=x(t+1)-x(t),
$$

for any sequence $\{x(t), t \in \mathbb{Z}\}$. For more details on the calculus of difference equation, we refer the reader to [10].

Clearly, the considered equation (1) has no nontrivial linear term. So, the inversion of such an equation needs some prepartions. More precisely, we have to transform the equation by adding a linear term to both sides in (1). Although the added term destroys a contraction already present but,

2010 Mathematics Subject Classification. Primray: 39A10, 39A12.

Key words and phrases. Krasnoselskii theorem, Fixed point, Periodic solutions, Large contraction, Difference equations. 
as we shall see, will replace it with the so called large contraction which is suitable in the fixed point theory. The integration gives rise to a fixed point mapping from which we define a compact operator and a large contraction. We prove that such a definition of maps fits very well to a nice modification of Krasnoselskii's fixed point theorem due T. A. Burton so that the existence of periodic solutions for equation (1) are readily obtained. For details on Krasnoselskii's theorem we refer the reader to [14] while full informations on the modification of Krasnoselskii theorem can be found in [5], [6] or [8]).

In section 2, we present the inversion of difference equations (1) and the modification of Krasnoselskii's fixed point theorem. We present our main results on periodicity in section 3 and at the end we provide an example to illustrate this work.

\section{INVERSION OF THE EQUATION}

Let $T>0$ be an integer such that $T \geq 1$. Define

$$
C_{T}:=\{\varphi \in C(\mathbb{Z}, \mathbb{R}): \varphi(t+T)=\varphi(t)\},
$$

where $C(\mathbb{Z}, \mathbb{R})$ is the space of all real valued functions. Then $\left(C_{T},\|\cdot\|\right)$ is a Banach space with the supremum norm

$$
\|\varphi\|:=\sup _{t \in[0, T-1]}|\varphi(t)| .
$$

We will assume that the following periodicity conditions hold.

$$
a(t+T)=a(t), c(t+T)=c(t), \tau(t+T)=\tau(t), \tau(t) \geq \tau^{*}>0
$$

for some constant $\tau^{*}$. Also, we assume that

$$
0<a(t)<1
$$

Further, we require that $q(t, x, y)$ is periodic in $t$ and Lipschitz continuous in $x$ and $y$. That is,

$$
q(t+T, x, y)=q(t, x, y)
$$

and there are positive constants $k_{1}, k_{2}$ such that

$$
|q(t, x, y)-q(t, z, w)| \leq k_{1}|x-z|+k_{2}|y-w|,
$$

for any $x, y, z, w \in \mathbb{R}$. 
Lemma 2.1. Suppose that $1-\prod_{s=t-T}^{t-1}(1-a(s)) \neq 0$, conditions (2) and (3) hold. Then $x \in C_{T}$ is a solution of equation (1) if and only if

$$
\begin{array}{r}
x(t)=c(t-1) x(t-\tau(t))+\left(1-\prod_{s=t-T}^{t-1}(1-a(s))\right)^{-1} \\
\cdot\left[\sum_{r=t-T}^{t-1} a(r)(x(r)-g(x(r))) \prod_{s=r+1}^{t-1}(1-a(s))\right. \\
+\sum_{r=t-T}^{t-1}[q(r, x(r), x(r-\tau(r))) \\
\left.-\mu(r) x(r-\tau(r))] \prod_{s=r+1}^{t-1}(1-a(s))\right]
\end{array}
$$

where $\mu(r)=c(r)-c(r-1)(1-a(r))$.

Proof. Let $x \in C_{T}$ be a solution of (1). First, write this equation as

$$
\begin{aligned}
\Delta x(t)+a(t) x(t)= & a(t) x(t)-a(t) g(x(t)) \\
& +c(t) \Delta x(t-\tau(t))+q(t, x(t), x(t-\tau(t))) .
\end{aligned}
$$

Multiplying both sides of the above equation by $\prod_{s=0}^{t}(1-a(s))^{-1}$ and then summing from $t-T$ to $t-1$, we obtain

$$
\begin{aligned}
& \sum_{r=t-T}^{t-1} \Delta\left[\prod_{s=0}^{r-1}(1-a(s))^{-1} x(r)\right] \\
& =\sum_{r=t-T}^{t-1}[a(r)\{x(r)-g(x(r))\}+c(r) \Delta x(r-\tau(r)) \\
& \quad+q(r, x(r), x(r-\tau(r)))] \prod_{s=0}^{r}(1-a(s))^{-1} .
\end{aligned}
$$

As a consequence, we arrive at

$$
\begin{aligned}
& \prod_{s=0}^{t-1}(1-a(s))^{-1} x(t)-\prod_{s=0}^{t-T-1}(1-a(s))^{-1} x(t-T) \\
& =\sum_{r=t-T}^{t-1}[a(r)\{x(r)-g(x(r))\}+c(r) \Delta x(r-\tau(r)) \\
& \quad+q(r, x(r), x(r-\tau(r)))] \prod_{s=0}^{r}(1-a(s))^{-1} .
\end{aligned}
$$


Now, by dividing both sides of the above equation by $\prod_{s=0}^{t-1}(1-a(s))^{-1}$ and using the fact $x(t)=x(t-T)$ we get

$$
\begin{aligned}
x(t)= & \left(1-\prod_{s=t-T}^{t-1}(1-a(s))\right)^{-1} \\
\times & \sum_{r=t-T}^{t-1}[a(r)\{x(r)-g(x(r))\}+c(r) \Delta x(r-\tau(r)) \\
& +q(r, x(r), x(r-\tau(r)))] \prod_{s=r+1}^{t-1}(1-a(s)) .
\end{aligned}
$$

But,

$$
\begin{aligned}
& \sum_{r=t-T}^{t-1} c(r) \Delta x(r-\tau(r)) \prod_{s=r+1}^{t-1}(1-a(s))=c(t-1) x(t-\tau(t)) \\
& \quad-c(t-T-1) x(t-T-\tau(t-T)) \prod_{s=t-T}^{t-1}(1-a(s)) \\
& \quad-\sum_{r=t-T}^{t-1} x(r-\tau(r)) \Delta\left[c(r-1) \prod_{s=r}^{t-1}(1-a(s))\right] .
\end{aligned}
$$

Thus, equation (7) becomes

$$
\begin{aligned}
& \left(1-\prod_{s=t-T}^{t-1}(1-a(s))\right)^{-1} \times\left[\sum_{r=t-T}^{t-1} a(r)\{x(r)-g(x(r))\} \prod_{s=r+1}^{t-1}(1-a(s))\right. \\
& \quad+\sum_{r=t-T}^{t-1} q(r, x(r), x(r-\tau(r))) \prod_{s=r+1}^{t-1}(1-a(s)) \\
& +\left(1-\prod_{s=t-T}^{t-1}(1-a(s))\right) c(t-1) x(t-\tau(t)) \\
& \left.\quad-\sum_{r=t-T}^{t-1} x(r-\tau(r)) \Delta\left(c(r-1) \prod_{s=r}^{t-1}(1-a(s))\right)\right] \\
& =c(t-1) \\
& \quad x(t-\tau(t))+\left(1-\prod_{s=t-T}^{t-1}(1+a(s))\right)^{-1} \\
& \times \sum_{r=t-T}^{t-1}[a(r)\{x(r)-g(x(r))\}-\mu(r) x(r-\tau(r))
\end{aligned}
$$




$$
+q(r, x(r), x(r-\tau(r)))] \prod_{s=r+1}^{t-1}(1-a(s)),
$$

where $\mu(r)=c(r)-c(r-1)(1-a(r))$.

As mentioned above, we employ, in our analysis, a fixed point theorem in which the notion of a large contraction is required as sufficient conditions. For that, we give the following definition which can be found in [8] or [6].

Definition 2.1 (Large contraction). Let $(M, d)$ be a metric space and $B$ : $M \rightarrow M$. $B$ is said to be a large contraction if $\varphi, \psi \in M$, with $\varphi \neq \psi$ then $d(B \varphi, B \psi)<d(\varphi, \psi)$ and if for all $\epsilon>0$ there exists $\delta<1$ such that

$$
[\varphi, \psi \in M, d(\varphi, \psi) \geq \epsilon] \Longrightarrow d(B \varphi, B \psi) \leq \delta d(\varphi, \psi) \text {. }
$$

Theorem 2.1. Let $(M, d)$ be a complete metric space and $B$ be a large contraction. Suppose there is an $x \in M$ and $L>0$, such that $d\left(x, B^{n} x\right) \leq L$ for all $n \geq 1$. Then $B$ has a unique fixed point in $M$.

The next theorem, which constitutes a basis for our main results, is a reformulated version of Krasnoselskii's fixed point theorem. This version is due to T. A. Burton (see [5], [6]).

Theorem 2.2 (Krasnoselskii-Burton). Let $M$ be a closed bounded convex non-empty subset of a Banach space $(S,\|\|$.$) . Suppose that A, B$ map $M$ into $M$ and that

(i) for all $x, y \in M \Longrightarrow A x+B y \in M$,

(ii) $A$ is continuous and $A M$ is contained in a compact subset of $M$,

(iii) $B$ is a large contraction.

Then there is a $z \in M$ with $z=A z+B z$.

We will use this theorem to prove the existence of periodic solutions for (1). We begin with the following result.

Theorem 2.3. Let $\|$.$\| be the supremum norm,$

$$
M:=\{\varphi \in C(\mathbb{Z}, \mathbb{R}),\|\varphi\| \leq L\},
$$

and define

$$
(F \varphi)(t):=\varphi(t)-g(\varphi(t)) .
$$

Suppose that $g$ is satisfying the following conditions

H1. $g: \mathbb{R} \rightarrow \mathbb{R}$ is continuous on $[-L, L]$ and differentiable on $(-L, L)$,

H2. The function $g$ is strictly increasing on $[-L, L]$,

H3. $\sup _{t \in(-L, L)} g^{\prime}(t) \leq 1$.

Then the mapping $F$ is a large contraction on the set $M$.

The proof of this theorem can be found in [1, Theorem $3.4 \mathrm{p} \mathrm{9].}$ 


\section{EXIsTENCE OF PERIODiC SOLUTIONS}

To apply Theorem 2.2, we need to define a Banach space $\mathfrak{P}$, a bounded convex subset $M$ of $\mathfrak{P}$ and construct two mappings, one is a large contraction and the other is compact. So, we let $(\mathfrak{P},\|\cdot\|)=\left(C_{T},\|\cdot\|\right)$ and $M=\{\varphi \in \mathfrak{P} \mid\|\varphi\| \leq L\}$, where $L$ is a positive constant. We express equation (6) as

$$
\varphi(t)=(\mathcal{B} \varphi)(t)+(A \varphi)(t):=(H \varphi)(t),
$$

where $A, \mathcal{B}: M \rightarrow \mathfrak{P}$ are defined by

$$
\begin{aligned}
& (A \varphi)(t)=c(t-1) \varphi(t-\tau(t))+\left(1-\prod_{s=t-T}^{t-1}(1-a(s))\right)^{-1} \\
& \times \sum_{r=t-T}^{t-1}[q(r, \varphi(r), \varphi(r-\tau(r)))-\mu(r) \varphi(r-\tau(r))] \prod_{s=r+1}^{t-1}(1-a(s)),
\end{aligned}
$$

and

$$
\begin{aligned}
(\mathcal{B} \varphi)(t)=(1 & \left.-\prod_{s=t-T}^{t-1}(1-a(s))\right)^{-1} \\
& \times \sum_{r=t-T}^{t-1} a(r)(\varphi(r)-g(\varphi(r))) \prod_{s=r+1}^{t-1}(1-a(s)) .
\end{aligned}
$$

Suppose further that the following hypotheses hold true

$$
\begin{gathered}
|\mu(t)| \leq \delta a(t), \\
\left(\left(k_{1}+k_{2}\right) L+|q(t, 0,0)|\right) \leq \beta L a(t), \\
\alpha=\max _{t \in[0, T]}|c(t-1)|, \\
J(\alpha+\delta+\beta) \leq 1,
\end{gathered}
$$

where $\alpha, \beta, J$ are constants with $J \geq 3$.

Now we have sufficient elements to prove that the mapping $H$ has a fixed point which solves (1).

Lemma 3.1. For A defined in (8), suppose that (2)-(5) and (10)-(13) hold then $A: M \rightarrow M$ is continuous in the maximum norm and maps $M$ into a compact subset of $M$.

Proof. We first show that $A: M \rightarrow M$. Let $\varphi \in M$. Evaluate (8) at $t+T$,

$$
(A \varphi)(t+T)=c(t+T-1) \varphi(t+T-\tau(t+T))+\left(1-\prod_{s=t}^{t+T-1}(1-a(s))\right)^{-1}
$$




$$
\times \sum_{r=t}^{t+T-1}[q(r, \varphi(r), \varphi(r-\tau(r)))-\mu(r) \varphi(r-\tau(r))] \prod_{s=r+1}^{t+T-1}(1-a(s)) .
$$

Let $j=r-T$, then

$$
\begin{aligned}
(A \varphi)(t+T)=c( & t+T-1) \varphi(t+T-\tau(t+T))+\left(1-\prod_{s=t}^{t+T-1}(1-a(s))\right)^{-1} \\
& \times \sum_{j=t-T}^{t-1}[q(j+T, \varphi(j+T), \varphi(j+T-\tau(j+T))) \\
& -\mu(j+T) \varphi(j+T-\tau(j+T))] \prod_{s=j+T+1}^{t+T-1}(1-a(s)) .
\end{aligned}
$$

Now, by (2) and letting $k=s-T$, we see that

$$
\begin{aligned}
& (A \varphi)(t+T)=c(t-1) \varphi(t-\tau(t))+\left(1-\prod_{k=t-T}^{t-1}(1-a(s))\right)^{-1} \\
& \quad \times \sum_{j=t-T}^{t-1}[q(j, \varphi(j), \varphi(j-\tau(j)))-\mu(j) \varphi(j-\tau(j))] \prod_{k=j+1}^{t-1}(1-a(k)) \\
& =(A \varphi)(t) .
\end{aligned}
$$

That is, $A: C_{T} \rightarrow C_{T}$.

In view of (5) we derive the following inequality

$$
\begin{aligned}
|q(t, x, y)| & =|q(t, x, y)-q(t, 0,0)+q(t, 0,0)| \\
& \leq|q(t, x, y)-q(t, 0,0)|+|q(t, 0,0)| \\
& \leq k_{1}\|x\|+k_{2}\|y\|+|q(t, 0,0)| .
\end{aligned}
$$

Note that from (3), we have $1-\prod_{k=t-T}^{t-1}(1-a(s))>0$. Consequently, having in mind conditions (10)-(13), we obtain for any $\varphi \in M$,

$$
\begin{aligned}
& |(A \varphi)(t)| \leq|c(t-1) \varphi(t-\tau(t))|+\left(1-\prod_{s=t-T}^{t-1}(1-a(s))\right)^{-1} \\
& \quad \times \sum_{r=t-T}^{t-1}[|\mu(r) \varphi(r-\tau(r))|+|q(r, \varphi(r), \varphi(r-\tau(r)))|] \prod_{s=r+1}^{t-1}(1-a(s)) \\
& \leq \alpha|\varphi(t-\tau(t))|+\left(1-\prod_{s=t-T}^{t-1}(1-a(s))\right)^{-1}
\end{aligned}
$$




$$
\begin{aligned}
& \times \sum_{r=t-T}^{t-1}\left[\delta a(r)|\varphi(r-\tau(r))|+\left(\left(k_{1}+k_{2}\right) L+|q(t, 0,0)|\right)\right] \prod_{s=r+1}^{t-1}(1-a(s)) \\
\leq & \alpha|\varphi(t-\tau(t))|+\left(1-\prod_{s=t-T}^{t-1}(1-a(s))\right)^{-1} \\
& \times \sum_{r=t-T}^{t-1} L[\delta+\beta] a(r) \prod_{s=r+1}^{t-1}(1-a(s)) \\
\leq & \alpha L+\left(1-\prod_{s=t-T}^{t-1}(1-a(s))\right)^{-1} \sum_{r=t-T}^{t-1} L[\delta+\beta] a(r) \prod_{s=r+1}^{t-1}(1-a(s)) \\
\leq & (\alpha+\delta+\beta) L \leq \frac{L}{J} \\
\leq & L .
\end{aligned}
$$

Thus $A \varphi \in M$.

Hence, $A$ maps $M$ into itself. That is $A: M \rightarrow M$.

Next we show that $A$ is continuous in the supremum norm. Let $\varphi, \psi \in M$, and let

Then,

$$
\eta:=\left(1-\prod_{s=t-T}^{t-1}(1-a(s))\right)^{-1}
$$

$$
\begin{aligned}
& |(A \varphi)(t)-(A \psi)(t)| \leq|c(t-1)||\varphi(t-\tau(t))-\psi(t-\tau(t))| \\
& \quad+\left(1-\prod_{s=t-T}^{t-1}(1-a(s))\right)^{-1} \sum_{r=t-T}^{t-1}[|\mu(r)||\varphi(r-\tau(r))-\psi(r-\tau(r))| \\
& \quad+q(r, \varphi(r), \varphi(r-\tau(r)))-q(r, \psi(r), \psi(r-\tau(r)))] \prod_{s=r+1}^{t-1}(1-a(s)) \\
& \leq \alpha\|\varphi-\psi\|+\delta\left(1-\prod_{s=t-T}^{t-1}(1-a(s))\right)^{-1} \sum_{r=t-T}^{t-1} a(r) \prod_{s=r+1}^{t-1}(1-a(s)) \\
& \quad+\left(k_{1}+k_{2}\right)\|\varphi-\psi\|\left(1-\prod_{s=t-T}^{t-1}(1-a(s))\right)^{-1} \sum_{r=t-T}^{t-1} \prod_{s=r+1}^{t-1}(1-a(s)) \\
& \leq\left(\alpha+\delta+\left(k_{1}+k_{2}\right) T \eta\right)\|\varphi-\psi\| .
\end{aligned}
$$

Let $\epsilon>0$ be arbitrary. Define $\gamma=\epsilon / K$ with $K=\alpha+\delta+\left(k_{1}+k_{2}\right) T \eta$, where $k_{1}, k_{2}$ are given by (5). Then, for $\|\varphi-\psi\| \leq \gamma$ we obtain

$$
\|A \varphi-A \psi\| \leq K\|\varphi-\psi\|<\epsilon .
$$

This proves that $A$ is continuous. 
Left to show that $A$ maps bounded subsets into compact sets. As $M$ is bounded and we have proved that $A$ is continuous and $A M$ is subset of $\mathbb{R}^{T}$ which is bounded. Thus $A M$ is contained in a compact subset of $M$. Therefore, $A$ is continuous in $M$ and $A M$ is contained in a compact subset of $M$.

In the next result we assume that for all $t \in \mathbb{R}$ and $\psi \in M$,

$$
\max (|F(L)|,|F(-L)|) \leq\left(\frac{J-1}{J}\right) L,
$$

where $(F \varphi)(t)=\varphi(t)-g(\varphi(t))$ is the one seen in $(7)$.

Lemma 3.2. Let $\mathcal{B}$ be defined by (9) and suppose that (2)-(3), (14) and all conditions of Proposition (2.3) hold. Then $\mathcal{B}: M \rightarrow M$ is a large contraction.

Proof. We first show that $\mathcal{B}: M \rightarrow M$.

Let $\varphi \in M$. Evaluate (9) at $t+T$.

$$
\begin{aligned}
(\mathcal{B} \varphi)(t+T)= & \left(1-\prod_{s=t}^{t+T-1}(1-a(s))\right)^{-1} \\
& \times \sum_{r=t}^{t+T-1} a(r)(\varphi(r+1)-g(\varphi(r+1))) \prod_{s=r+1}^{t+T-1}(1-a(s)) .
\end{aligned}
$$

Let $j=r-T$, then

$$
\begin{aligned}
(\mathcal{B} \varphi) & (t+T)=\left(1-\prod_{s=t}^{t+T-1}(1-a(s))\right)^{-1} \\
& \times \sum_{j=t-T}^{t-1} a(j+T)(\varphi(j+T)-g(\varphi(j+T))) \prod_{s=j+T+1}^{t+T-1}(1-a(s)) .
\end{aligned}
$$

Now let $k=s-T$, then

$$
\begin{aligned}
(\mathcal{B} \varphi)(t+T)= & \left(1-\prod_{k=t-T}^{t-1}(1-a(s))\right)^{-1} \\
& \times \sum_{j=t-T}^{t-1} a(j)(\varphi(j)-g(\varphi(j))) \prod_{k=j+1}^{t-1}(1-a(k)) \\
= & (\mathcal{B} \varphi)(t) .
\end{aligned}
$$

That is, $\mathcal{B}: C_{T} \rightarrow C_{T}$. 
Note that from (3), we have $1-\prod_{s=t-T}^{t-1}(1-a(s))>0$. So, for any $\varphi \in M$, we have

$$
\begin{aligned}
|(\mathcal{B} \varphi)(t)| \leq & \left(1-\prod_{s=t-T}^{t-1}(1-a(s))\right)^{-1} \\
& \times \sum_{r=t-T}^{t-1} a(r)|\varphi(r)-g(\varphi(r))| \prod_{s=r+1}^{t-1}(1-a(s)) \\
\leq & \left(1-\prod_{s=t-T}^{t-1}(1-a(s))\right)^{-1} \\
& \times \sum_{r=t-T}^{t-1} a(r) \max (|F(L)|,|F(-L)|) \prod_{s=r+1}^{t-1}(1-a(s)) \\
\leq & \left(\frac{J-1}{J}\right) L\left(1-\prod_{s=t-T}^{t-1}(1-a(s))\right)^{-1} \sum_{r=t-T}^{t-1} a(r) \prod_{s=r+1}^{t-1}(1-a(s)) \\
\leq & L .
\end{aligned}
$$

Thus $\mathcal{B} \varphi \in M$. Consequently, we have $\mathcal{B}: M \rightarrow M$. It remains to show that $\mathcal{B}$ is a large contraction with a unique fixed point in $M$. From Theorem 2.3 we know that $\varphi(t)-g(\varphi(t))$ is a large contraction in the maximum norm. For any $\epsilon$, let $\varsigma<1$ be the constant found for $\varphi(t)-g(\varphi(t))$. Then,

$$
\begin{aligned}
&\|\mathcal{B} \varphi-\mathcal{B} \psi\| \leq\left(1-\prod_{s=t-T}^{t-1}(1-a(s))\right)^{-1} \\
& \times \sum_{r=t-T}^{t-1} a(r) \varsigma\|\varphi-\psi\| \prod_{s=r+1}^{t-1}(1-a(s)) \\
& \leq \varsigma\|\varphi-\psi\| .
\end{aligned}
$$

Thus, $\|\mathcal{B} \varphi-\mathcal{B} \psi\| \leq \varsigma\|\varphi-\psi\|$. Consequently, $\mathcal{B}$ is a large contraction.

Theorem 3.1. Let $\left(C_{T},\|\|.\right)$ be the Banach space of continuous T-periodic real valued functions and $M=\left\{\varphi \in C_{T},\|\varphi\| \leq L\right\}$, where $L$, is positive constant. Suppose (2)-(3), (10)-(14) and all conditions of Theorem 2.3 hold. Then, equation (1) prossesses a T-periodic solution lying in the subset $M$.

Proof. By Lemma 3.1, $A: M \rightarrow M$ is continuous and $A M$ is contained in a compact set. Also, from Lemma 3.2, the mapping $\mathcal{B}: M \rightarrow M$ is a large contraction. Moreover, making use of (14), we see that if $\varphi, \psi \in M$, then

$$
\|A \varphi+\mathcal{B} \psi\| \leq\|A \varphi\|+\|\mathcal{B} \psi\|
$$




$$
\leq \frac{L}{J}+\left(\frac{J-1}{J}\right) L \leq L
$$

Thus $A \varphi+\mathcal{B} \psi \in M$.

Clearly, all hypotheses of Krasnoselskii-Burton's theorem are satisfied. Thus, there exists a fixed point $\varphi \in M$ such that $\varphi=A \varphi+\mathfrak{B} \varphi$. Hence (1) has a $\mathrm{T}$ - periodic solution in $M$.

\section{EXAMPLE}

We consider the totally nonlinear difference equation with functional delay

$$
\begin{aligned}
\Delta x(t)=- & 0.0536 x^{3}(t)+2.10^{-2} \cos (t) \Delta x(t-\tau(t)) \\
& +10^{-4}(\sin (x(t))+\cos (x(t-\tau(t)))), \quad t \in \mathbb{Z},
\end{aligned}
$$

where

$$
\tau(t+T)=\tau(t)
$$

So, we have

$$
\begin{gathered}
a(t)=0.536, \quad c(t)=2.10^{-2} \cos (t), \quad g(x)=x^{3}, \quad L=\sqrt{3} / 3, \\
q(t, x, y)=10^{-4}(\sin (x)+\cos (y)) .
\end{gathered}
$$

Clearly, $q(t, x, y)$ is periodic in $t$ and Lipschitz continuous in $x$ and $y$. That is

$$
q(t+T, x, y)=q(t, x, y)
$$

and

$$
\begin{aligned}
|q(t, x, y)-q(t, z, w)| & =\left|10^{-4}(\sin (x)-\sin (z)+\cos (y)-\cos (w))\right| \\
& \leq\left|10^{-4}(\sin (x)-\sin (z))\right|+\left|10^{-4}(\cos (y)-\cos (w))\right| \\
& \leq 10^{-4}|x-z|+10^{-4}|y-w| .
\end{aligned}
$$

We have

$$
\begin{aligned}
\alpha & =2.10^{-2} \\
|\mu(t)| & =\left|2.10^{-2} \cos (t)-2.10^{-2}(1-0.536) \cos (t-1)\right| \\
& \leq 0.122 \leq 0.02 \times 0.536 \leq \delta a(t), \quad \forall t \in \mathbb{Z},
\end{aligned}
$$

and

$$
\begin{aligned}
\left(\left(k_{1}+k_{2}\right) L+|q(t, 0,0)|\right) & =2.10^{-4}(\sqrt{3} / 3)+10^{-4} \\
& \leq 0.16(0.536 \times(\sqrt{3} / 3)) \\
& \leq \beta L a(t), \quad \forall t \in \mathbb{Z} .
\end{aligned}
$$

By substituting $\alpha, \beta, \delta$ in (13), we obtain $J \in[3,5]$. Define $M=\{\varphi \in$ $\left.C_{T} \mid\|\varphi\| \leq L\right\}$, where $L=\sqrt{3} / 3$. Then, the difference equation (15) possesses, by Theorem 3.1, a $T$-periodic solution in $M$. 


\section{REFERENCES}

[1] M. Adivar and Y. N. Raffoul, Existence of periodic solutions in totally nonlinear delay dynamic equations, Electronic Journal of Qualitative Theory of Differential Equations, 2009, No. 1, 1-20.

[2] A. Ardjouni and A. Djoudi, Periodic solution in totally nonlinear dynamic equations with functional delay on a time scale, Rend. Sem. Mat. Univ. Politec.Torino, Vol.68, 4 (2010), 349-359.

[3] A. Ardjouni and A. Djoudi, Existence and positivity of solutions for totally nonlinear neutral periodic differential equation, Miskolc Mathematical Notes Vol. 14 (2013), No. 3, pp. $757-768$.

[4] A. Ardjouni and A. Djoudi, Periodic solutions in totally nonlinear difference equations with functional delay, Stud. Univ. Babes-Bolyai Math, 56 (2011). No. 3, 7-17.

[5] T. A. Burton, Liapunov functionals, fixed points and stability by Krasnoselskii's theorem, Nonlinear Stud. 9(2002), No. 2, 181-190.

[6] T. A. Burton, Stability by fixed point Theory for Functional Differential Equations, Dover Publications, NewYork, 2006.

[7] T. A. Burton, fixed point theorem of Krasnoselskii, App. Math. Lett. 11 (1998), 85-88.

[8] T. A. Burton, Integral equations, implicit relations and fixed points, Proc. Amer. Math. Soc. 124 (1996); 2383-2390.

[9] H. Deham and A. Djoudi, Periodic solutions for nonlinear differential equation whith functional delay, Georgian Mathematical Journal, 15 (2008), No. 4, 635-642.

[10] W. G. Kelly, A.C. Peterson, Difference Equations: An Introduction with Applications, Academic Press, 2001.

[11] Y. M. Dib, M. R. Maroun and Y.N. Raffoul, Periodicity and stability in neutral nonlinear differential equations whith functional delay, Electronic Journal of Differential Equations, Vol 2005 (2005), No. 142, pp. 1-11.

[12] Y. N. Raffoul, Stability and periodicity in discrete delay equations, J. Math. Anal. App., 324(2006), no. 2, 1356-1362.

[13] Y. N. Raffoul, Periodicity in general delay non-linear difference equations using fixed point theory, J. Difference Equ. App., 10(2004), no. 13-15, 1329-1242.

[14] D. R. Smart, Fixed point theorems, Cambridge Tracts in Mathematics, No. 66. Cambridge University Press, London-New York, 1974. 


\section{IMENe SOUALHiA}

Applied Mathematics Lab

FACUlty of Sciences

Department of Mathematics

Univ Annaba

P.O. Box 12, Annaba 23000

Algeria

E-mail address: magistere1983@yahoo.fr

\section{Abdelouaheb Ardjouni}

Faculty of Sciences and Technology

Department of Mathematics and Informatics Univ Souk Ahras

P.O. Box 1553, Souk Ahras, 41000

Algeria

Address 2:

Applied Mathematics Lab

Faculty of Sciences

Department of Mathematics

UnIV AnNABA

P.O. Box 12, Annaba 23000

Algeria

E-mail address: abd_ardjouni@yahoo.fr

\section{Ahcene Duoudi}

Applied Mathematics Lab

FACUlty of Sciences

Department of Mathematics

Univ Annaba

P.O. Box 12, Annaba 23000

Algeria

E-mail address: adjoudi@yahoo.com 